PROCEEDINGS OF THE

AMERICAN MATHEMATICAL SOCIETY

Volume 133, Number 1, Pages 203-212

S 0002-9939(04)07556-2

Article electronically published on June 18, 2004

\title{
CONVERGENCE IN ALMOST PERIODIC COOPERATIVE SYSTEMS WITH A FIRST INTEGRAL
}

\author{
WENXIAN SHEN AND XIAO-QIANG ZHAO \\ (Communicated by Carmen C. Chicone)
}

\begin{abstract}
This paper is to investigate the asymptotic dynamics in almost periodic cooperative systems with a first integral. By appealing to the theory of skew-product semiflows we establish the asymptotic almost periodicity of bounded solutions to such systems, which extends the existing convergence results for time independent and periodic cooperative systems with a first integral and proves a conjecture of B. Tang, Y. Kuang and H. Smith in SIAM J. Math. Anal., 24 (1993), 1331-1339.
\end{abstract}

\section{INTRODUCTION}

Monotone dynamics has received extensive investigations (see, e.g., 2, 5, 10. 14, 15, 16, 19 and references therein). Various stability and convergence results were obtained for autonomous and periodic monotone systems under appropriate assumptions. Some of these results have also been extended to the almost periodic/nonautonomous case by using the powerful skew-product flows approach (see, e.g., [4, 14, 18, and references therein). The current paper is devoted to the study of convergence in almost periodic cooperative systems with a first integral. The convergence of positive bounded solutions in time independent and periodic as well as some special almost periodic cooperative systems with a first integral has been discussed in several papers. For example, Nakajima [9] proved the asymptotic periodicity of bounded solutions for periodic and cooperative gross-substitute systems with a linear first integral, and this convergence result was further generalized to the almost periodic case by Sell and Nakajima [13. Mierczynski [8] proved the convergence of bounded solutions for autonomous and strictly cooperative systems with a first integral, and Jiang [6] obtained the same result in the case where these systems are cooperative. In 1993, Tang, Kuang and Smith [17] considered strictly cooperative systems on $\mathbb{R}_{+}^{n}$ with a general class of first integrals in $\mathbb{R}_{+}^{n}$, and conjectured that every bounded solution converges to an almost periodic solution. Meanwhile, they partly proved this conjecture under some additional conditions on the first integral. In 1996, Jiang [7] gave an affirmative answer to this conjecture in the case where these systems are periodic and cooperative. It should be mentioned

Received by the editors June 17, 2003 and, in revised form, September 24, 2003.

2000 Mathematics Subject Classification. Primary 34C12, 34C27, 37B55.

Key words and phrases. Cooperative systems, first integral, almost periodic solutions, skewproduct semiflows.

The first author's research was supported in part by NSF grant DMS-0103381.

The second author's research was supported in part by the NSERC of Canada. 
that Arino and his colleagues also studied asymptotic behavior in nonautonomous delay differential equations and monotone semiflows with a monotone first integral (see [1] and the references therein).

The purpose of this paper is to prove Tang, Kuang and Smith's conjecture for the almost periodic and cooperative case by appealing to the theory of skew-product semiflows. Differently from [17, we establish the uniform stability of nonnegative solutions and the 1-cover property of the associated omega limit sets of the skew-product semiflow without using the Liapunov function method. The paper is organized as follows. In the next section we present some basic definitions and our main result and give a simple but illustrative example. In Section 3 we prove the main result and make two remarks on it.

\section{Definitions And THE MAIN RESUlt}

For $x=\left(x_{1}, \cdots, x_{n}\right) \in \mathbb{R}^{n}$, we define $\|x\|=\max _{1 \leq i \leq n}\left|x_{i}\right|$. A function $f \in$ $C\left(\mathbb{R}, \mathbb{R}^{n}\right)$ is said to be almost periodic if for any $\epsilon>0$, there exists $l=l(\epsilon)>0$ such that every interval of $\mathbb{R}$ of length $l$ contains at least one point of the set $T(\epsilon):=\{\tau \in \mathbb{R}:\|f(t+\tau)-f(t)\|<\epsilon, \forall t \in \mathbb{R}\}$. Let $D \subset \mathbb{R}^{n}$ be a closed set. A function $f \in C\left(\mathbb{R} \times D, \mathbb{R}^{n}\right)$ is said to be uniformly almost periodic in its first variable if $f(\cdot, x)$ is almost periodic for each $x \in D$, and for any compact set $E \subset D$, $f$ is uniformly continuous on $\mathbb{R} \times E$. Let $f \in C\left(\mathbb{R} \times D, \mathbb{R}^{n}\right)$ be uniformly almost periodic in its first variable. For given $\lambda \in \mathbb{R}$ and $x \in D$, define

$$
a(f, \lambda, x)=\lim _{T \rightarrow \infty} \frac{1}{T} \int_{0}^{T} f(t, x) e^{-i \lambda t} d t .
$$

The set

$$
\operatorname{Exp}(f)=\{\lambda \in \mathbb{R}: a(f, \lambda, x) \not \equiv 0 \text { on } D\}
$$

is called the set of exponents of $f$, and the module of $f, \mathcal{M}(f)$, is defined as the smallest additive group of real numbers containing $\operatorname{Exp}(f)$.

Let $\left(Y, d_{Y}\right)$ be a compact metric space with metric $d_{Y}$. A continuous flow $\sigma$ : $Y \times \mathbb{R} \rightarrow Y$ is said to be minimal if $Y$ contains no nonempty, proper, closed invariant subset; distal if for any two distinct points $y_{1}$ and $y_{2}$ in $Y$, it follows that $\inf _{t \in \mathbb{R}} d_{Y}\left(\sigma\left(y_{1}, t\right), \sigma\left(y_{2}, t\right)\right)>0$. Thus, a flow $\sigma: Y \times \mathbb{R} \rightarrow Y$ is minimal if and only if every full orbit is dense in $Y$.

Let $\mathbb{R}_{+}^{n}=\left\{\left(x_{1}, \cdots, x_{n}\right) \in \mathbb{R}^{n}: x_{i} \geq 0, \forall 1 \leq i \leq n\right\}$. For $x=\left(x_{1}, \cdots, x_{n}\right) \in \mathbb{R}^{n}$ and $y=\left(y_{1}, \cdots, x_{n}\right) \in \mathbb{R}^{n}$, we write $x \leq y$ if $y-x \in \mathbb{R}_{+}^{n}, x<y$ if $y-x \in$ $\mathbb{R}_{+}^{n} \backslash\{0\}$, and $x \ll y$ if $y-x \in \operatorname{Int}\left(\mathbb{R}_{+}^{n}\right)$. Let $K \subset \mathbb{R}^{n}$ be a compact subset of $\mathbb{R}^{n}$, and for each $1 \leq i \leq n$, define $b_{* i}=\min \left\{x_{i}: x=\left(x_{1}, \cdots, x_{i}, \cdots, x_{n}\right) \in K\right\}$ and $b_{i}^{*}=\max \left\{x_{i}: x=\left(x_{1}, \cdots, x_{i}, \cdots, x_{n}\right) \in K\right\}$. Then $b_{*}=\left(b_{* 1}, b_{* 2}, \cdots, b_{* n}\right)$ and $b^{*}=\left(b_{1}^{*}, b_{2}^{*}, \cdots, b_{n}^{*}\right)$ are the greatest lower bound and least upper bound of $K$ with respect to the componentwise order $\leq$ on $\mathbb{R}^{n}$, respectively.

Consider the $n$-dimensional system of ordinary differential equations

$$
\dot{x}=F(t, x),
$$

where $F: \mathbb{R} \times \mathbb{R}_{+}^{n} \rightarrow \mathbb{R}^{n}$. We make the following standing assumptions throughout the paper.

(H1) $F \in C^{1}\left(\mathbb{R} \times \mathbb{R}_{+}^{n}, \mathbb{R}^{n}\right)$ is uniformly almost periodic in $t$, and for any compact subset $K$ of $\mathbb{R}_{+}^{n}$, the derivative $D_{x} F(t, x)$ is bounded on $\mathbb{R} \times K$; 
(H2) $F(t, 0) \geq 0$ and $\frac{\partial F_{i}}{\partial x_{j}} \geq 0$ for all $(t, x) \in \mathbb{R} \times \mathbb{R}_{+}^{n}, 1 \leq i \neq j \leq n$;

(H3) There is a $C^{1}$-function $H: \mathbb{R}_{+}^{n} \rightarrow \mathbb{R}$ such that $\operatorname{grad} H(x) \gg 0$ at each $x \in \mathbb{R}_{+}^{n}$, and $\langle\operatorname{grad} H(x), F(t, x)\rangle=0$ for all $(t, x) \in \mathbb{R} \times \mathbb{R}_{+}^{n}$.

Clearly, (H1) guarantees the local existence and uniqueness of solutions of the almost periodic system (2.1) with initial values in $\mathbb{R}_{+}^{n} \cdot(\mathrm{H} 2)$ implies that $\mathbb{R}_{+}^{n}$ is positively invariant for system (2.1), and that (2.1) is a cooperative (quasimonotone) system and hence, the comparison principle holds. (H3) says that $H(x)$ has positive gradient at each $x \in \mathbb{R}_{+}^{n}$ and is a first integral of system (2.1), i.e., $H(x)$ is a constant along every solution of (2.1).

Denote $\phi(t ; x, F)$ as the solution of (2.1) with $\phi(0 ; x, F)=x \in \mathbb{R}_{+}^{n}$. Let $\phi(t ; x, F)$ be defined for all $t \geq 0$. Then $\phi(t ; x, F)$ is said to be asymptotic to an almost periodic solution if there is an almost periodic solution $\phi^{*}(t ; F)$ of (2.1) such that

$$
\lim _{t \rightarrow \infty}\left(\phi(t ; x, F)-\phi^{*}(t ; F)\right)=0 ;
$$

$\phi(t ; x, F)$ is said to be uniformly stable if for any $\epsilon>0$, there is $\delta=\delta(\epsilon)>0$ such that

$$
\|\phi(t+\tau ; y, F)-\phi(t+\tau ; x, F)\| \leq \epsilon, \quad \forall t \geq 0
$$

whenever $\tau>0$ and $y \in \mathbb{R}_{+}^{n}$ with $\|\phi(\tau ; y, F)-\phi(\tau ; x, F)\| \leq \delta$.

Let

$$
\mathcal{F}=\operatorname{cl}\left\{F_{\tau}: \tau \in \mathbb{R}\right\},
$$

where $F_{\tau}(t, x)=F(t+\tau, x)$ and the closure is taken under the open compact topology. Define $\sigma(t)(G)=G_{t}=G(\cdot+t, \cdot), \forall t \in \mathbb{R}, G \in \mathcal{F}$. Then $\sigma(t): \mathcal{F} \rightarrow \mathcal{F}$ is a compact, almost periodic minimal and distal flow (see [12, Section VI.C]). By assumption (H2), each $G \in \mathcal{F}$ is a continuous function on $\mathbb{R} \times \mathbb{R}_{+}^{n}$ with $G(t, 0) \geq$ $0, \forall t \in \mathbb{R}$. Moreover, (H1) and (H2) imply that $G$ is locally Lipschitz in $x \in \mathbb{R}_{+}^{n}$, and $G_{i}(t, x) \leq G_{i}(t, y)$ for any $0 \leq x \leq y$ and $1 \leq i \leq n$ with $x_{i}=y_{i}$. It then follows that system (2.1) induces the following (local) skew-product flow:

$$
\begin{gathered}
\Pi_{t}: \mathbb{R}_{+}^{n} \times \mathcal{F} \rightarrow \mathbb{R}_{+}^{n} \times \mathcal{F}, \\
\Pi_{t}(x, G)=\left(\phi(t ; x, G), G_{t}\right),
\end{gathered}
$$

where $\phi(t ; x, G)$ is the solution of

$$
\dot{x}=G(t, x)
$$

with $\phi(0 ; x, G)=x$. Furthermore, $\Pi_{t}$ is monotone in the sense that for any $x^{1}$, $x^{2} \in \mathbb{R}_{+}^{n}$ with $x^{1} \leq x^{2}$ and $G \in \mathcal{F}$,

$$
\phi\left(t ; x^{1}, G\right) \leq \phi\left(t ; x^{2}, G\right)
$$

for $t \geq 0$ at which both $\phi\left(t ; x^{1}, G\right)$ and $\phi\left(t ; x^{2}, G\right)$ exist.

Let $P: \mathbb{R}_{+}^{n} \times \mathcal{F} \rightarrow \mathcal{F}$ be the natural projection, i.e., $P(x, G)=G, \forall(x, G) \in$ $\mathbb{R}_{+}^{n} \times \mathcal{F}$. Recall that the omega limit set of a point $(x, G) \in \mathbb{R}_{+}^{n} \times \mathcal{F}$ with respect to $\Pi_{t}$ is defined as

$$
\omega(x, G)=\left\{(y, K) \in \mathbb{R}_{+}^{n} \times \mathcal{F}: \exists t_{n} \rightarrow \infty \text { such that } \Pi_{t_{n}}(x, G) \rightarrow(y, K)\right\} .
$$

A compact invariant set $E \subset \mathbb{R}_{+}^{n} \times \mathcal{F}$ of $\Pi_{t}$ is called a 1-cover of $\mathcal{F}$ if for any $G \in \mathcal{F}$, $\operatorname{card}\left(P^{-1}(G) \cap E\right)=1$. 
Now we are in a position to state our main result.

Theorem A. Assume that (H1), (H2) and (H3) hold. Then the following two statements are valid:

(1) If $\phi(t ; x, F)$ is a bounded solution of (2.1) in $\mathbb{R}_{+}^{n}$, then $\omega(x, F)$ is a 1-cover of $\mathcal{F}$;

(2) Every bounded solution $\phi(t ; x, F)$ of (2.1) in $\mathbb{R}_{+}^{n}$ is asymptotic to an almost periodic solution $\phi^{*}(t ; F)$. Moreover, $\mathcal{M}\left(\phi^{*}\right) \subset \mathcal{M}(F)$.

Observe that, following from the module containment properties of almost periodic functions (see [3, Theorem 4.5]), it can be easily proved that if $F(t, x)$ is actually periodic in $t, F(t+T, x)=F(t, x)$, then Theorem A (2) implies that every bounded solution of (2.1) in $\mathbb{R}_{+}^{n}$ is asymptotic to a periodic solution of period $T$.

At the end of this section we use a simple example, which is motivated by [7], to illustrate our main result.

Example. Consider the following two-dimensional system on $\mathbb{R}_{+}^{2}$ :

$$
\begin{aligned}
& \dot{x}_{1}=a(t) x_{2} e^{x_{1}}-e^{x_{1}}+1, \\
& \dot{x}_{2}=1-e^{-x_{1}}-a(t) x_{2},
\end{aligned}
$$

where $a(t)$ is a nonnegative, almost periodic $C^{1}$-function on $\mathbb{R}$. It is easy to verify that system (2.5) admits a first integral $H\left(x_{1}, x_{2}\right)=1-e^{-x_{1}}+x_{2}$ on $\mathbb{R}_{+}^{2}$, and that (H1)-(H3) hold. Thus, Theorem A implies that every bounded solution of (2.5) in $\mathbb{R}_{+}^{2}$ is asymptotic to an almost periodic solution. We should point out that the main result [17, Theorem 3.1] cannot be applied to the almost periodic system (2.5) since the Liapunov function associated with $H\left(x_{1}, x_{2}\right)$,

$$
V(x, y)=\left|e^{-x_{1}}-e^{-y_{1}}\right|+\left|x_{2}-y_{2}\right|, \quad \forall x=\left(x_{1}, x_{2}\right), y=\left(y_{1}, y_{2}\right) \in \mathbb{R}_{+}^{2},
$$

does not satisfy the condition that $\lim _{|x-y| \rightarrow+\infty} V(x, y)=+\infty$.

\section{Proof of Theorem A}

In order to prove Theorem A, we need a series of lemmas.

Lemma 3.1. If $\phi(t ; x, F)$ is a bounded solution of (2.1) in $\mathbb{R}_{+}^{n}$, then it is uniformly stable.

Proof. Let $e=(1,1, \cdots, 1) \in \operatorname{Int} \mathbb{R}_{+}^{n}$. By the boundedness of $\phi(t ; x, F)$, we can choose a sufficiently large real number $r>0$ such that

$$
0 \leq \phi(t ; x, F) \leq q_{0}:=r e, \quad \forall t \geq 0 .
$$

Define

$$
M=\max _{1 \leq i \leq n}\left\{\max _{0 \leq x \leq q_{0}+e} H_{x_{i}}(x)\right\}, \quad m=\min _{1 \leq i \leq n}\left\{\min _{0 \leq x \leq q_{0}+e} H_{x_{i}}(x)\right\} .
$$

Then (H3) implies that $M, m>0$. Note that

$$
H(y)-H(z)=\sum_{i=1}^{n} \int_{0}^{1} H_{x_{i}}(z+s(y-z)) d s \cdot\left(y_{i}-z_{i}\right), \quad \forall y, z \in \mathbb{R}_{+}^{n} .
$$


It follows that

$$
|H(y)-H(z)| \leq n M\|y-z\|, \quad \forall 0 \leq z, y \leq q_{0}+e,
$$

and

$$
|H(y)-H(z)| \geq m\|y-z\|, \quad \forall 0 \leq z \leq y \leq q_{0}+e .
$$
that

$$
\phi(\tau ; x, F)-\delta(\epsilon) e \leq \phi(\tau ; x, F) \leq \phi(\tau ; x, F)+\delta(\epsilon) e \leq q_{0}+e
$$

for all $\tau \geq 0$. Let

$$
p(\epsilon, \tau)=\left(\max \left(\phi_{1}(\tau ; x, F)-\delta(\epsilon), 0\right), \cdots, \max \left(\phi_{n}(\tau ; x, F)-\delta(\epsilon), 0\right)\right)
$$

and $q(\epsilon, \tau)=\phi(\tau ; x, F)+\delta(\epsilon) e$. Since

$$
0 \leq q(\epsilon, \tau)-p(\epsilon, \tau)=[\phi(\tau ; x, F)-\delta(\epsilon) e]-p(\epsilon, \tau)+2 \delta(\epsilon) e \leq 2 \delta(\epsilon) e
$$

for all $\tau \geq 0$, (3.2) and (3.4) imply that

$$
|H(p(\epsilon, \tau))-H(q(\epsilon, \tau))| \leq n M \epsilon, \quad \forall \tau \geq 0 .
$$

For given $\tau \geq 0$, let

$$
U(\epsilon, \tau)=\left\{z \in \mathbb{R}_{+}^{n}: p(\epsilon, \tau) \leq z \leq q(\epsilon, \tau)\right\} .
$$

Clearly, $\phi(\tau ; x, F) \in U(\epsilon, \tau)$. Let $[0, \sigma)$ be the maximal interval of existence of the solution $\phi\left(t ; q(\epsilon, \tau), F_{\tau}\right)$. By the comparison principle, we then have

$$
\phi\left(t ; p(\epsilon, \tau), F_{\tau}\right) \leq \phi\left(t ; \phi(\tau ; x, F), F_{\tau}\right) \leq \phi\left(t ; q(\epsilon, \tau), F_{\tau}\right)
$$

and

$$
\phi\left(t ; p(\epsilon, \tau), F_{\tau}\right) \leq \phi\left(t ; z, F_{\tau}\right) \leq \phi\left(t ; q(\epsilon, \tau), F_{\tau}\right)
$$

for all $t \in[0, \sigma)$ and $z \in U(\epsilon, \tau)$. By (H3) and (3.5),

$$
\begin{aligned}
\left|H\left(\phi\left(t ; q(\epsilon, \tau), F_{\tau}\right)\right)-H\left(\phi\left(t ; \phi(\tau ; x, F), F_{\tau}\right)\right)\right| & =|H(q(\epsilon, \tau))-H(\phi(\tau ; x, F))| \\
& \leq|H(q(\epsilon, \tau))-H(p(\epsilon, \tau))| \\
& \leq n M \epsilon
\end{aligned}
$$

for all $t \in[0, \sigma)$. By (3.3) and (3.4), we have

$$
\left\|\phi\left(t ; q(\epsilon, \tau), F_{\tau}\right)-\phi\left(t ; \phi(\tau ; x, F), F_{\tau}\right)\right\| \leq \frac{n M}{m} \epsilon
$$

for all $t \in[0, \sigma)$ with $\phi\left(t ; q(\epsilon, \tau), F_{\tau}\right) \in\left[0, q_{0}+e\right]$. We claim that

$$
\phi\left(t ; q(\epsilon, \tau), F_{\tau}\right) \in\left[0, q_{0}+e\right], \quad \forall t \in[0, \sigma) .
$$

Otherwise, there exists a real number $t^{*} \in(0, \sigma)$ such that $\phi\left(t ; q(\epsilon, \tau), F_{\tau}\right) \in$ $\left[0, q_{0}+e\right], \forall t \in\left[0, t^{*}\right]$ and $\left\|\phi\left(t^{*} ; q(\epsilon, \tau), F_{\tau}\right)\right\|=\left\|q_{0}\right\|+1$. But the inequality (3.6) implies that

$$
\begin{aligned}
\left\|\phi\left(t^{*} ; q(\epsilon, \tau), F_{\tau}\right)\right\| \leq & \left\|\phi\left(t^{*} ; \phi(\tau ; x, F), F_{\tau}\right)\right\| \\
& \quad+\left\|\phi\left(t^{*} ; q(\epsilon, \tau), F_{\tau}\right)-\phi\left(t^{*} ; \phi(\tau ; x, F), F_{\tau}\right)\right\| \\
\leq & \left\|q_{0}\right\|+\frac{n M}{m} \epsilon_{0} \leq\left\|q_{0}\right\|+\frac{1}{2}
\end{aligned}
$$


a contradiction. It then follows that $\sigma=\infty$ and for every $z \in U(\epsilon, \tau)$, the solution $\phi\left(t ; z, F_{\tau}\right)$ exists globally on $[0, \infty)$ and

$$
\phi\left(t ; z, F_{\tau}\right) \in\left[0, q_{0}+e\right], \quad \forall t \geq 0 .
$$

Similarly, we can show that for any $z \in U(\epsilon, \tau)$,

$$
\left\|\phi\left(t ; q(\epsilon, \tau), F_{\tau}\right)-\phi\left(t ; z, F_{\tau}\right)\right\| \leq \frac{n M}{m} \epsilon, \quad \forall t \geq 0 .
$$

Then (3.6) and (3.7) imply that for any $z \in U(\epsilon, \tau)$ and $t \geq 0$,

$$
\begin{aligned}
\left\|\phi\left(t ; \phi(\tau ; x, F), F_{\tau}\right)-\phi\left(t ; z, F_{\tau}\right)\right\| \leq & \left\|\phi\left(t ; \phi(\tau ; x, F), F_{\tau}\right)-\phi\left(t ; q(\epsilon, \tau), F_{\tau}\right)\right\| \\
& +\left\|\phi\left(t ; q(\epsilon, \tau), F_{\tau}\right)-\phi\left(t ; z, F_{\tau}\right)\right\| \\
\leq & \frac{n M}{m} \epsilon+\frac{n M}{m} \epsilon=\frac{2 n M}{m} \epsilon .
\end{aligned}
$$

For any $\epsilon \in\left(0, \epsilon_{0}\right], \tau \geq 0$, and $y \in \mathbb{R}_{+}^{n}$ with $\|\phi(\tau ; y, F)-\phi(\tau ; x, F)\| \leq \delta(\epsilon)$, we have $\phi(\tau ; y, F) \in U(\epsilon, \tau)$. Then the inequality (3.8) with $z=\phi(\tau ; y, F)$ implies that

$$
\begin{aligned}
\|\phi(t+\tau ; y, F)-\phi(t+\tau ; x, F)\| & \left.=\| \phi\left(t ; \phi(\tau ; y, F), F_{\tau}\right)-\phi\left(t ; \phi(\tau ; x, F), F_{\tau}\right)\right) \| \\
& \leq \frac{2 n M}{m} \epsilon, \quad \forall t \geq 0 .
\end{aligned}
$$

Thus, $\phi(t ; x, F)$ is uniformly stable.

Lemma 3.2. If $\phi(t ; x, F)$ is a bounded and uniformly stable solution of (2.1) in $\mathbb{R}_{+}^{n}$, then $\Pi_{t}: \omega(x, F) \rightarrow \omega(x, F)$ is a minimal and distal flow, and for any $(y, G) \in$ $\omega(x, F), \tilde{G} \in \mathcal{F}$ and any sequence $\left\{t_{i}\right\} \subset \mathbb{R}$ with $\tilde{G}_{t_{i}} \rightarrow G$, there is a sequence $\left\{\left(y^{i}, \tilde{G}\right)\right\} \subset \omega(x, F)$ such that $\phi\left(t_{i} ; y^{i}, \tilde{G}\right) \rightarrow y$.

Proof. The minimality and distality follow from [11, Theorem 5] (see also [14, Theorem II.2.8]). The last statement is implied by [14, Lemma I.2.16 and Theorem I.2.6], as applied to the epimorphism of minimal and distal flows $P:(\omega(x, F), \Pi(t)) \rightarrow$ $(\mathcal{F}, \sigma(t))$.

Lemma 3.3. Suppose that $\phi(t ; x, F)$ is a bounded solution of (2.1) in $\mathbb{R}_{+}^{n}$. Let $p(G)$ and $q(G)$ be the greatest lower bound and least upper bound of the set $\{y \in$ $\left.\mathbb{R}_{+}^{n}:(y, G) \in \omega(x, F)\right\}$, respectively. Then

$$
\phi\left(t ; p\left(G_{-t}\right), G_{-t}\right)=p(G), \quad \phi\left(t ; q\left(G_{-t}\right), G_{-t}\right)=q(G)
$$

for all $t \geq 0$, and for any $t_{n} \rightarrow \infty$ with $G_{t_{n}} \rightarrow G\left(G_{-t_{n}} \rightarrow G\right)$, we have $p\left(G_{t_{n}}\right) \rightarrow$ $p(G)\left(p\left(G_{-t_{n}}\right) \rightarrow p(G)\right)$.

Proof. We only prove the lemma for $p(\cdot)$ since a similar argument applies to $q(\cdot)$.

First, for any $G \in \mathcal{F}$ and $(y, G) \in \omega(x, F)$, we have $p(G) \leq y$, and hence

$$
\phi(t ; p(G), G) \leq \phi(t ; y, G), \quad \forall t \geq 0 .
$$

Since $\Pi_{t} \omega(x, F)=\omega(x, F)$ for all $t \in \mathbb{R}$, we then obtain

$$
\phi(t ; p(G), G) \leq p\left(G_{t}\right) \leq \phi(t ; y, G), \quad \forall t \geq 0,(y, G) \in \omega(x, F) .
$$

For given $G \in \mathcal{F}$, let $t_{n} \rightarrow \infty$ be such that

$$
\left(\phi\left(t_{n} ; p(G), G\right), G_{t_{n}}\right) \rightarrow(\tilde{p}, G), \quad p\left(G_{t_{n}}\right) \rightarrow p^{*} .
$$


We prove that $p^{*}=p(G)=\tilde{p}$. In fact, by Lemma 3.2 for any $(y, G) \in \omega(x, F)$, there is $\left(y^{n}, G\right) \in \omega(x, F)$ such that

$$
\phi\left(t_{n} ; y^{n}, G\right) \rightarrow y
$$

as $n \rightarrow \infty$. It then follows from (3.9) and (3.10) that

$$
\tilde{p} \leq p^{*} \leq p(G) .
$$

By (H3), we have $H(\tilde{p})=H(p(G))$, and hence

$$
\tilde{p}=p^{*}=p(G) .
$$

Next, since $\phi\left(t ; \phi\left(t_{n} ; p(G), G\right), G_{t_{n}}\right)=\phi\left(t+t_{n} ; p(G), G\right)$, (3.12) implies that

$$
\phi(t ; p(G), G)=\lim _{n \rightarrow \infty} \phi\left(t+t_{n} ; p(G), G\right)
$$

for all $t \in \mathbb{R}$. By (3.9) it follows that for any $(y, G) \in \omega(x, F)$ and $t \in \mathbb{R}$,

$$
\phi\left(t+t_{n} ; p(G), G\right) \leq \phi\left(t+t_{n} ; y, G\right) \text { for } n \gg 1 .
$$

By similar arguments as in proving (3.9) and (3.11), it follows that

$$
\phi(t ; p(G), G) \leq p\left(G_{t}\right), \quad \forall t \in \mathbb{R} .
$$

Using (3.9), we then have

$$
p(G)=\phi\left(t ; \phi(-t ; p(G), G), G_{-t}\right) \leq \phi\left(t ; p\left(G_{-t}\right), G_{-t}\right) \leq p(G), \quad \forall t \geq 0 .
$$

This implies that

$$
\phi\left(t ; p\left(G_{-t}\right), G_{-t}\right)=p(G), \quad \forall t \geq 0 .
$$

Now, for given $G \in \mathcal{F}$, suppose that $t_{n} \rightarrow \infty$ is such that $G_{-t_{n}} \rightarrow G$ as $n \rightarrow \infty$. Without loss of generality, assume that $p\left(G_{-t_{n}}\right) \rightarrow p^{* *}$ as $n \rightarrow \infty$. We are to prove that $p^{* *}=p(G)$. Using Lemma 3.2 again, we obtain

$$
p^{* *} \leq p(G) \text {. }
$$

By (3.14) and (H3), $H\left(p\left(G_{-t}\right)\right)=H(p(G))$ for $t \geq 0$. Therefore, we have

$$
H\left(p^{* *}\right)=\lim _{n \rightarrow \infty} H\left(p\left(G_{-t_{n}}\right)\right)=H(p(G)) .
$$

It then follows from (3.15), 3.16), and (H3) that $p^{* *}=p(G)$.

Lemma 3.4. Let $x^{1}, x^{2} \in \mathbb{R}_{+}^{n}$ with $x^{1} \leq x^{2}$. If $x_{i_{0}}^{1}<x_{i_{0}}^{2}$ for some $1 \leq i_{0} \leq n$, then

$$
\phi_{i_{0}}\left(t ; x^{1}, F\right)<\phi_{i_{0}}\left(t ; x^{2}, F\right)
$$

for each $t>0$ at which both $\phi\left(t ; x^{1}, F\right)$ and $\phi\left(t ; x^{2}, F\right)$ exist.

Proof. Let $\xi(t)=\phi_{i_{0}}\left(t ; x^{2}, F\right)-\phi_{i_{0}}\left(t ; x^{1}, F\right)$. By (H2), it follows that

$$
\begin{aligned}
\dot{\xi}(t)= & F_{i_{0}}\left(t, \phi\left(t ; x^{2}, F\right)\right)-F_{i_{0}}\left(t, \phi\left(t ; x^{1}, F\right)\right) \\
= & \sum_{i=1}^{n} \int_{0}^{1} \frac{\partial F_{i_{0}}}{\partial x_{i}}\left(t, \phi\left(t ; x^{1}, F\right)+s\left(\phi\left(t ; x^{2}, F\right)-\phi\left(t ; x^{1}, F\right)\right)\right) d s \\
& \cdot\left(\phi_{i}\left(t ; x^{2}, F\right)-\phi_{i}\left(t ; x^{1}, F\right)\right) \\
\geq & \int_{0}^{1} \frac{\partial F_{i_{0}}}{\partial x_{i_{0}}}\left(t, \phi\left(t ; x^{1}, F\right)+s\left(\phi\left(t ; x^{2}, F\right)-\phi\left(t ; x^{1}, F\right)\right)\right) d s \cdot \xi(t) .
\end{aligned}
$$


Define

$$
b(t)=\int_{0}^{1} \frac{\partial F_{i_{0}}}{\partial x_{i_{0}}}\left(t, \phi\left(t ; x^{1}, F\right)+s\left(\phi\left(t ; x^{2}, F\right)-\phi\left(t ; x^{1}, F\right)\right)\right) d s .
$$

We then have

$$
\xi(t) \geq e^{\int_{0}^{t} b(s) d s} \xi(0)>0, \quad \forall t \geq 0,
$$

which implies that $\phi_{i_{0}}\left(t ; x^{1}, F\right)<\phi_{i_{0}}\left(t ; x^{2}, F\right)$.

Proof of Theorem A. (1) Let $\phi(t ; x, F)$ be a bounded solution of (2.1) in $\mathbb{R}_{+}^{n}$. By Lemmas 3.1 and 3.2, $\Pi_{t}: \omega(x, F) \rightarrow \omega(x, F)$ is a minimal and distal flow. Let $p(F)$ be the greatest lower bound of the set $\left\{y \in \mathbb{R}_{+}^{n}:(y, F) \in \omega(x, F)\right\}$. It follows that for each $1 \leq i \leq n$, there is $\left(y^{i}, F\right) \in \omega(x, F)$ such that

$$
y_{i}^{i}=p_{i}(F) \text {. }
$$

By Lemma 3.3 we have

$$
\phi\left(t ; p\left(F_{-t}\right), F_{-t}\right)=p(F), \quad \forall t \geq 0 .
$$

Note that

$$
p\left(F_{-t}\right) \leq \phi\left(-t ; y^{i}, F\right)
$$

and

$$
\phi_{i}\left(t ; p\left(F_{-t}\right), F_{-t}\right)=p_{i}(F)=y_{i}^{i}=\phi_{i}\left(t ; \phi\left(-t ; y^{i}, F\right), F_{-t}\right)
$$

for all $t \geq 0$. By Lemma 3.4

$$
p_{i}\left(F_{-t}\right)=\phi_{i}\left(-t ; y^{i}, F\right), \quad \forall t \geq 0 .
$$

By Lemma 3.2 it follows that for any $(y, F) \in \omega(x, F)$, there is $t_{n} \rightarrow \infty$ such that

$$
\left(\phi\left(-t_{n} ; y^{i}, F\right), F_{-t_{n}}\right) \rightarrow(y, F)
$$

Using Lemma 3.3 again, we obtain

$$
p\left(F_{-t_{n}}\right) \rightarrow p(F) .
$$

By (3.17)-(3.19), it then follows that

$$
y_{i}=p_{i}(F), \quad \forall(y, F) \in \omega(x, F), 1 \leq i \leq n .
$$

Therefore, $y=p(F)$ for any $(y, F) \in \omega(x, F)$, and hence, $\operatorname{card}\left(P^{-1}(F) \cap \omega(x, F)\right)=$ 1. By the structure theorem of skew-product flows [11, Theorem 1], as applied to the flow $\Pi_{t}: \omega(x, F) \rightarrow \omega(x, F)$, it follows that $\operatorname{card}\left(P^{-1}(G) \cap \omega(x, F)\right)=1$ for any $G \in \mathcal{F}$. Thus, $\omega(x, F)$ is a 1 -cover of $\mathcal{F}$.

(2) Let $\phi(t ; x, F)$ be a bounded solution of 2.1 in $\mathbb{R}_{+}^{n}$. By $(1), \omega(x, F)$ is a 1cover of $\mathcal{F}$. It then follows that $\Pi_{t}: \omega(x, F) \rightarrow \omega(x, F)$ is a compact, almost periodic minimal and distal flow. Let $\left\{\left(x^{*}, F\right)\right\}=P^{-1}(F) \cap \omega(x, F)$. Then $\phi\left(t ; x^{*}, F\right)$ is almost periodic in $t$ (see [12, Lemma VI.9]). It is easy to see that

$$
\lim _{t \rightarrow \infty}\left(\phi(t ; x, F)-\phi\left(t ; x^{*}, F\right)\right)=0 .
$$

Thus, $\phi(t ; x, F)$ is asymptotic to the almost periodic solution $\phi\left(t ; x^{*}, F\right)$. Since $\omega(x, F)$ is a 1-cover of $\mathcal{F}$, for any $\left\{\alpha_{n}\right\} \subset \mathbb{R}$ with $\lim _{n \rightarrow \infty} F\left(t+\alpha_{n}, x\right)=F(t, x)$ for $(t, x) \in \mathbb{R} \times \mathbb{R}_{+}^{n}$, we must have $\lim _{n \rightarrow \infty} \phi\left(t+\alpha_{n} ; x^{*}, F\right)=\phi\left(t ; x^{*}, F\right)$ for $t \in \mathbb{R}$. Then by [3, Theorem 4.5], $\mathcal{M}\left(\phi\left(\cdot ; x^{*}, F\right)\right) \subset \mathcal{M}(F)$.

Remark 3.1. By the proof of Theorem A, it is easy to see that if (H1), (H2) and (H3) hold on $\mathbb{R} \times \mathbb{R}^{n}$, then Theorem $\mathrm{A}$ is valid for the almost periodic system (2.1) defined on $\mathbb{R}^{n}$. 
Remark 3.2. Assume that (H1), (H2) and the following (H3)' hold:

$(\mathrm{H} 3)^{\prime}$ There is a $C^{1}$-function $H: \mathbb{R}_{+}^{n} \rightarrow \mathbb{R}$ such that $\operatorname{grad} H(x) \gg 0$ at each $x \in \operatorname{Int}\left(\mathbb{R}_{+}^{n}\right)$, and $\langle\operatorname{grad} H(x), F(t, x)\rangle=0$ for all $(t, x) \in \mathbb{R} \times \mathbb{R}_{+}^{n}$.

Then the conclusions of Theorem $\mathrm{A}$ are valid for any bounded solution $\phi(t ; x, F)=$ $\left(\phi_{1}(t ; x, F), \cdots, \phi_{n}(t ; x, F)\right.$ with $\liminf _{t \rightarrow \infty} \phi_{i}(t ; x, F)>0, \forall 1 \leq i \leq n$. Indeed, in this case we have $\omega(x, F) \subset \operatorname{Int}\left(\mathbb{R}_{+}^{n}\right) \times \mathcal{F}$. Then there exist $t_{0} \geq 0, p_{0} \gg 0$ and $q_{0} \gg 0$ such that $\phi(t ; x, F) \geq p_{0}, \forall t \geq t_{0}$, and $\omega(x, F) \subset\left[p_{0}, q_{0}\right] \times \mathcal{F}$. Let $(\bar{x}, \bar{F})=\Pi_{t_{0}}(x, F)$. Then $\omega(\bar{x}, \bar{F})=\omega(x, F)$ and $\phi\left(t+t_{0} ; x, F\right)=\phi(t ; \bar{x}, \bar{F}), \forall t \geq 0$. Without loss of generality, we may assume that $t_{0}=0$ (otherwise we replace $(x, F)$ with $(\bar{x}, \bar{F}))$. By assumption $(\mathrm{H} 3)^{\prime}$, it easily follows that $H(u)>H(v)$ for all $u \in \operatorname{Int}\left(\mathbb{R}_{+}^{n}\right)$ and $v \in \mathbb{R}_{+}^{n}$ with $u>v$. Thus, the proofs of Lemmas 3.1 and 3.3, and hence the proof of Theorem $\mathrm{A}$, still apply to $\phi(t ; x, F)$.

\section{REFERENCES}

[1] O. Arino, Monotone semi-flows which have a monotone first integral, in "Delay Differential Equations and Dynamical Systems", Lecture Notes in Math. 1475, Springer-Verlag, Berlin/Heidelberg, 1991, pp. 64-75. MR93a:34081

[2] X.-Y. Chen and H. Matano, Convergence, asymptotic periodicity, and finite-point blow-up in one-dimensional semilinear heat equations, J. Diff. Eqs., 78 (1989), 160-190. MF 90e:35018

[3] A. M. Fink, "Almost Periodic Differential Equations", Lecture Notes in Mathematics 377, Springer-Verlag, Berlin/Heidelberg/New York, 1974. MR 57:792

[4] G. Hetzer and W. Shen, Uniform persistence, coexistence, and extinction in almost periodic/nonautonomous competition diffusion systems, SIAM J. Math. Anal., 34 (2002), 204227. MR2003m:37143

[5] M. Hirsch, Stability and convergence in strongly monotone dynamical systems, J. Reine Angew. Math., 383 (1988), 1-53. MR:89c:58108

[6] J.-F. Jiang, Type $K$-monotone systems with an order-increasing invariant function, Chinese Ann. Math., Ser. B, 17 (1996), 335-342. MR97g:34062

[7] J.-F. Jiang, Periodic monotone systems with an invariant function, SIAM J. Math. Anal., 27 (1996), 1738-1744. MR98h:34089

[8] J. Mierczynski, Strictly cooperative systems with a first integral, SIAM J. Math. Anal., 18 (1987), 642-646. MR88e:34093

[9] F. Nakajima, Periodic time-dependent gross-substitute systems, SIAM J. Appl. Math., 36 (1979), 421-427. MR80c:93045

[10] P. Polacik, Convergence in smooth strongly monotone flows defined by semilinear parabolic equations, J. Diff. Eqs., 79 (1989), 89-110. MR90f:58025

[11] R. J. Sacker and G. R. Sell, "Lifting Properties in Skew-Product Flows with Applications to Differential Equations", Memoirs Amer. Math. Soc. , No. 190, Vol. 11, Providence, R.I., 1977. MR $56: 6632$

[12] G. Sell, "Topological Dynamics and Ordinary Differential Equations", Van Nostrand Reinhold, London, 1971. MR.56:1283

[13] G. R. Sell and F. Nakajima, Almost periodic time-dependent gross-substitute dynamical systems, Tohoku Math. J., 32 (1980), 255-263. MR81j:34075

[14] W. Shen and Y. Yi, "Almost Automorphic and Almost Periodic Dynamics in Skewproduct Semiflows", Memoirs Amer. Math. Soc., No. 647, Vol. 136, Providence, R.I., 1998. MR 99d:34088

[15] H. L. Smith, "Monotone Dynamical Systems, An Introduction to the Theory of Competitive and Cooperative Systems", Mathematical Surveys and Monographs, Vol. 41, Amer. Math. Soc., Providence, RI, 1995. MR96c:34002

[16] P. Takac, Asymptotic behavior of strongly monotone time-periodic dynamical processes with symmetry, J. Diff. Eqs., 100 (1992), 355-378. MR94d:47060

[17] B. Tang, Y. Kuang and H. Smith, Strictly nonautonomous cooperative system with a first integral, SIAM J. Math. Anal., 24 (1993), 1331-1339. MR94g:34067 
[18] X.-Q. Zhao, Global attractivity in monotone and subhomogeneous almost periodic systems, J. Differential Equations, 187 (2003), 494-509.

[19] X.-Q. Zhao, "Dynamical Systems in Population Biology", CMS books in Mathematics, Vol. 16, Springer-Verlag, New York, 2003.

Department of Mathematics, Auburn University, Auburn, Alabama 36849

E-mail address: ws@math. auburn.edu

Department of Mathematics and Statistics, Memorial University of Newfoundland, St. John's, Newfoundland, Canada A1C 5S7

E-mail address: xzhao@math.mun.ca 